computer-assisted segmentation of intestinal polyps to help doctors visualise polyps in images.

Methods We choose DCNN (Deep Convolution Neural Network) as our model, which has shown outstanding performance in image segmentation tasks in recent years. The data we used to train the model including two open intestinal polyp datasets, CVC-ColonDB and CVC-ClinicDB. The data are divided into training data, validation data and test data. At the same time, we use cross-entropy as a model-optimised loss function and the IoU value as our model's evaluation criteria. Results Table 1 shows that our model performed well on these three data. At the same time, it should be noted that our model performed significantly better on the training data than the test data as shown in IDDF2018-ABS-0259 Figure 1. This may be due to the lack of data and the variance between different patients.

Abstract IDDF2018-ABS-0259 Table 1 loU value of two classes (background and polyp) on the separated dataset

\begin{tabular}{lccc}
\hline DATA & $\begin{array}{c}\text { Background } \\
\text { loU }\end{array}$ & $\begin{array}{c}\text { Polyp } \\
\text { loU }\end{array}$ & Accuracy \\
\hline Training & 0.976 & 0.800 & 0.987 \\
data & & & 0.970 \\
Valid data & 0.969 & 0.604 & 0.725 \\
Test data & 0.947 & 0.523 & \\
\hline
\end{tabular}

Conclusions The experimental results show that our model has achieved good performance in the polyp segmentation task, which appears as an IoU value of more than 0.5 on the test set and visual examples illustrated in Figure 1. In addition, without the need for manual feature extraction and preprocessing, DCNN model shows good adaptation for polyps of different sizes.

\section{IDDF2018-ABS-0260 DEEP LEARNING FOR POLYP SEGMENTATION}

${ }^{1}$ Liansheng Wang*, ${ }^{1}$ Cong Xie, ${ }^{2}$ Yanxing Hu. ${ }^{1}$ Xiamen University, China; ${ }^{2}$ Xiamen Innovision Medical Technology Company, China

\subsection{6/gutjnl-2018-IDDFabstracts. 181}

Background Colorectal cancer is one of the commonest malignancies which threaten the health of human. Most of the colorectal cancers arise from adenomatous polyps, screening for this cancer is effective not only for early detection but also for prevention. Endoscopic examination is an important way to reveal polyps. At present, the main limitation of colonoscopy is the high polyp miss rate. This study aims to improve the accuracy of detection and save doctors' time with deep learning techniques.

Methods The data we used is a combination of CVCColonDB and CVC-ClinicDB which contains 912 images with associated polyps. We treat the rest part of a colonoscopy image except polys as background. Convolutional networks are applied to polyp localization and segmentation. There are three different architectures which include FCN, SegNet and modified ResNet. We trained convolutional networks with these architectures respectively and evaluated these models based on Intersection over Union (IoU) and accuracy.

Results The segmentation results on the test set are reported in table 1 and the accuracy of three architectures is greater than 90\%. Results achieved in SegNet and modified ResNet are very encouraging, with a IoU higher than $50 \%$ for polyps. IDDF2018-ABS-0260 Figure 1 shows qualitative results of three architectures. It can be seen that all of them recognise the location of polyp successfully and rows 2 and 3 show finer boundaries. The results are reported on test set

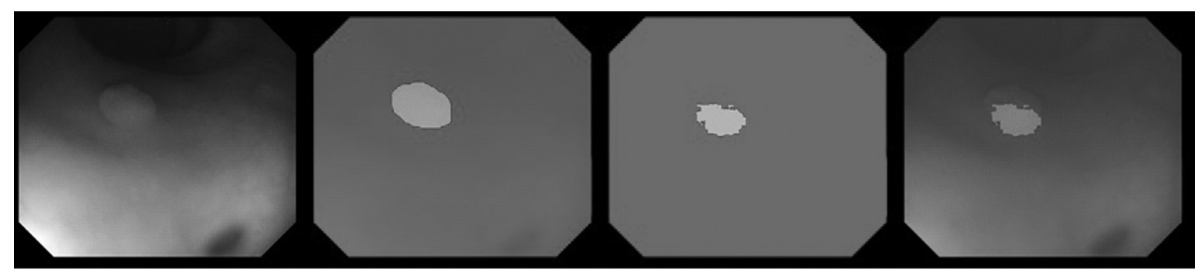

(a)

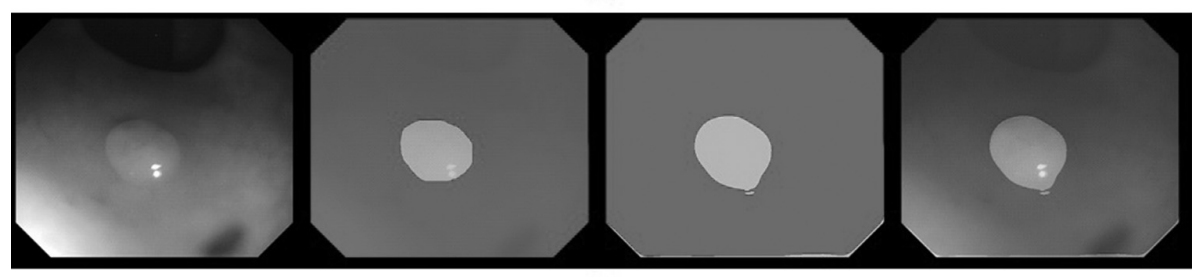

(b)

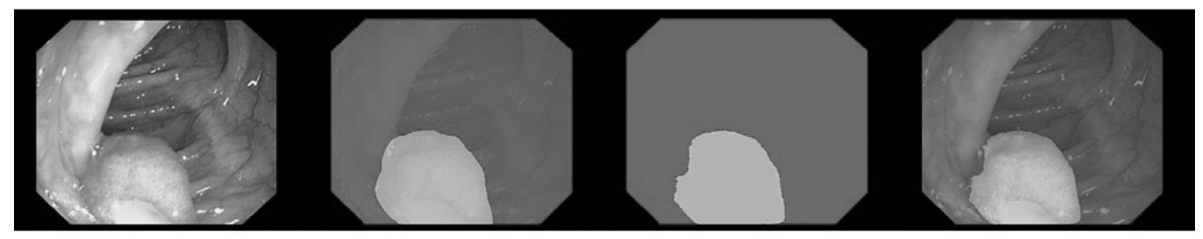

(c)

Abstract IDDF2018-ABS-0260 Figure 1 Examples of predictions: (a) FCN8 model; (b) SegNet model; (c) modified ResNet model. From left to right, each column represents ground truth, prediction and a translucent prediction image 
Abstract IDDF2018-ABS-0260 Table 1 Endoluminal scene semantic segmentation results for different architectures

\begin{tabular}{lcccc}
\hline Architecture & loU background & loU polyp & IoU mean & Acc mean \\
\hline FCN8 & 94.024 & 46.99 & 70.51 & 94.32 \\
SegNet & 94.897 & 53.795 & 74.346 & 95.183 \\
ResNet with changes & 96.029 & 64.0307 & 80.0299 & 96.291 \\
\hline
\end{tabular}

Conclusions Compare to traditional time-consuming handcrafted segmentation methods, when considering polyp segmentation, approaches based on deep learning are time-saving and effective, showing good results in colonoscopy images. Given that three architectures we mentioned above not only performs well but also allows for nearly real-time processing, it has a great potential in polyp localization and segmentation.

\section{IDDF2018-ABS-0261 POLYP DETECTION USING AN UNET BASED MODEL}

${ }^{1}$ Liansheng Wang*, ${ }^{1}$ Rongzhen Chen, ${ }^{2}$ Yanxing Hu. ${ }^{1}$ Xiamen University, China; ${ }^{2}$ Xiamen Innovision Medical Technology Company, China

\subsection{6/gutjnl-2018-IDDFabstracts.182}

Background Polyp is an important cause of Colorectal cancer. Wireless Capsule Endoscopy(WCE) has been widely used in direct inspection of the gastrointestinal tract without any surgical operation. Doctors need screen twenty thousand pictures per patient manually, which is time-consuming and tedious work. Recently, Deep Convolutional Neural Networks (DCNNs) shows state of the art performance in various highlevel vision tasks. Therefore we present a computer-aided diagnosis model which utilises Unet to detect polyp automatically.

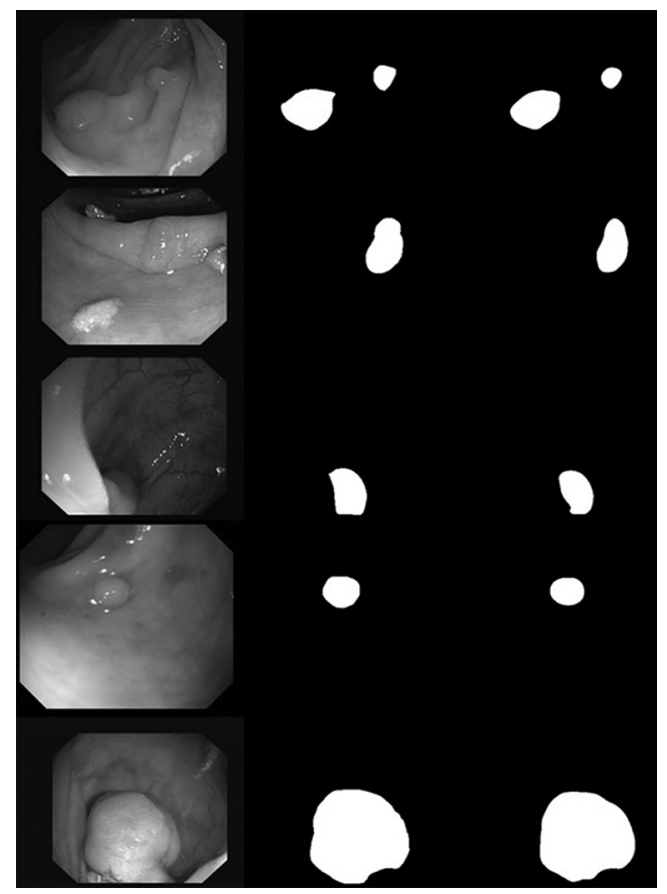

Abstract IDDF2018-ABS-0261 Figure 1 Segmentation results. Columns from left to right: origin image, ground truth, segmentation result
Methods Unet is proposed for biomedical image segmentation in recently years, showing the start of art result. In preprocessing stage, we utilise a weighted average filter to remove light spots covering polyps. Our model contains three downscale blocks and three upscale blocks. The downscale module consists of two $3 * 3$ conv layers and a max-pooling layer which captures high-dimensional characteristics and reduces the feature maps size. In every upscale module, we add a bilinear up-sampling layer to recover the spatial information. In addition, the upscale module's output is connected to previous downscale module's output, which promotes integration between low-level features and high-level features and accelerates the convergence of model. In the end, we use a median filter to remove small mistake response region caused by the poor environment in the gastrointestinal tract and fill small holes using morphology.

Results We train and test our model on CVC dataset that contains pixel-level polyp segmentation label. The dataset are divided into 656 (train), 169 (validation), and 87 (test) images. The results are evaluated in terms of pixel intersection-overunion (IOU). Our method finally obtains $73.91 \%$ on IOU and operates at 23.25 fps (Frames Per Second) that is far faster than screening manually. As shown in figure 1, the result is good enough to locate polyps.

Conclusions The result shows that our method can provide efficient and accurate assistance in the diagnosis of the digestive tract, which greatly reduces the workload of doctors. It thus has a potential to apply to clinical examination.

\section{IDDF2018-ABS-0262 PRIMARY INTESTINAL LYMPHANGIECTASIA IN YOUNG ADULT - A RARE CAUSE OF PROTEIN LOSING ENTEROPATHY}

Jayeeta Bhowmick*, Rajib Sarkar, Gopal Krishna Dhali. School of Digestive and Liver Diseases, Institute of Postgraduate Medical Education and Research, Kolkata, India

\subsection{6/gutjnl-2018-IDDFabstracts. 183}

Background Primary intestinal lymphangiectasia is a rare disorder characterised by dilated intestinal lacteals, causing lymph leakage into bowel lumen leading to lymphopenia, hypoalbuminemia, hypogammaglobulinemia, protein-losing enteropathy and chronic diarrhoea. It is generally diagnosed before 3 years of age, rarely presenting in adolescence and early adulthood. Usual features are bipedal oedema, serosal effusion, fatiguability, diarrhoea, recurrent infections. Differential diagnoses include constrictive pericarditis, Crohn's disease, intestinal tuberculosis, sarcoidosis and intestinal lymphoma. The gold standard for diagnosis is by histopathology of intestinal mucosa showing multiple dilated lacteals. Here we report a case of intestinal lymphangiectasia with a typical presentation like PLE and recurrent bacterial and fungal infection.

Methods On careful history elicitation and clinical examination, our patient was a 19-year-old male student, a resident of rural West Bengal, born of non-consanguineous parents, with chief complaints of bipedal swelling without oliguria and chronic small bowel type non-bloody diarrhoea since 5 years of age. Recurrent lower respiratory tract infections are leading to recurrent hospitalizations since his 1 month of age. No past history or contact with tuberculosis. He was short stature with BMI of 16 , no peripheral lymphadenopathy or organomegaly. 